Fusheng You

Xuetao Shi

Wanjun Shuai

Hongyi Zhang

Wei Zhang

Feng Fu

Ruigang Liu

Canhua Xu

Tingyi Bao

Xiuzhen Dong

\section{Applying electrical impedance tomography to dynamically monitor retroperitoneal bleeding in a renal trauma patient}

Accepted: 28 February 2013

Published online: 29 March 2013

(C) The Author(s) 2013. This article is published with open access at Springerlink.com

Dear Editor,

Active retroperitoneal bleeding (ARB) is characterized by a large amount of bleeding over a long period of time. Massive hemorrhage from $\mathrm{ARB}$, if not detected early enough, may lead to serious consequences including kidney resection and even death $[1,2]$. But up to now there has been no effective clinical imaging tool to continuously monitor patients and identify ARB. We present the first case report of the use of electrical impedance tomography (EIT), a noninvasive, dynamic and functional imaging modality, in the monitoring of ARB under clinical conditions.

A 27-year-old man was diagnosed with grade IV injury to the left kidney by $\mathrm{CT}$ and $\mathrm{B}$-scan ultrasonography, and was put on close clinical observation as conservative treatment. As well as conventional monitoring of vital signs, including blood pressure (BP), heart rate (HR) and $\mathrm{SpO}_{2}$, the patient was also continuously monitored by EIT for $9 \mathrm{~h}$.

The patient was kept lying supine quietly and 16 disposable $\mathrm{Ag}-\mathrm{AgCl}$ electrodes were attached around the body $2 \mathrm{~cm}$ below the injury and covering the area of the kidney. The electrodes were connected to an elastic belt to ensure good contact with the skin. The area of the injury, the electrodes and the elastic belt are shown in Fig. 1. The EIT data acquisition system was developed by the FMMU EIT Group [3-5]. A stimulus frequency of $50 \mathrm{kHz}$, a constant driving current of $1 \mathrm{~mA}$, the polar driving and adjacent measurement mode, and an imaging speed of one frame per second were applied. An optimized EIT image reconstruction strategy was applied using a damped least-squares method to improve the spatial resolution of the reconstructed images [5]. The reconstructed dynamic EIT images were integrated with one of the patient's CT images which acted as the static background image.

The results of the urine test confirmed gross hematuria and renal injury. The results of the blood tests were within normal ranges except that the WBC was high. There were no significant changes in vital signs (BP, $\mathrm{HR}$, and $\mathrm{SpO}_{2}$ ).

The fused EIT/CT images during the $9 \mathrm{~h}$ of EIT monitoring are shown in Fig. 2. At about 1330 hours the local image in the left renal region began to become red because of a decrease in impedance caused by the ARB. Up to about 1730 hours, the region showing the impedance decrease was almost fixed over the left renal area, and gradually deepened in color and increased in size. As the EIT monitoring showed a continuous decrease in resistivity and $\mathrm{ARB}$, a CT scan was performed, immediately followed by digital subtraction angiography (DSA) of the celiac artery and the renal artery. The DSA results confirmed the presence of ARB, and then an embolization operation was performed. B-scan ultrasonography after 2 days and a CT scan after 30 days showed the success of the operation. This implies that EIT, as a unique image

Fig. 1 Photographs showing the area of the injury, the EIT electrodes and the belt

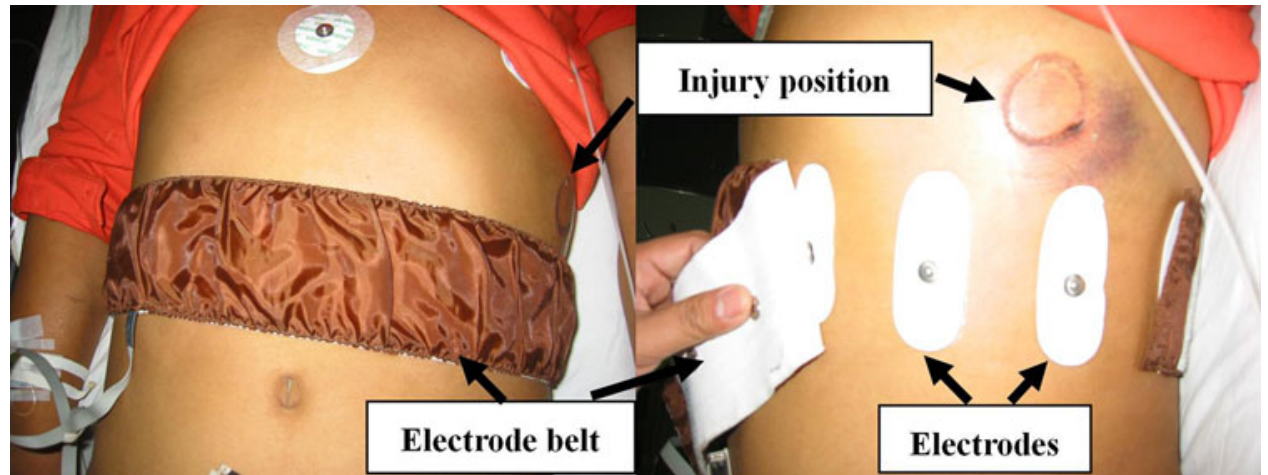




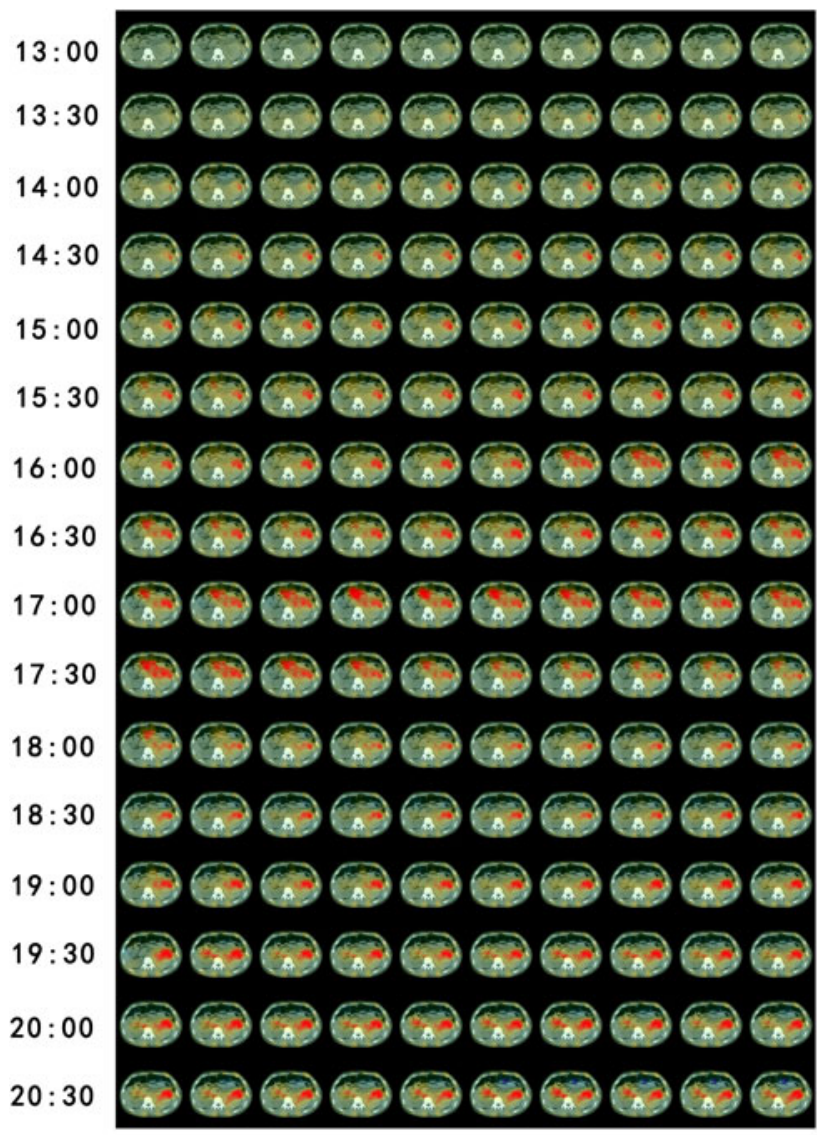

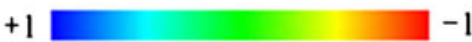

Fig. 2 Dynamic EIT monitoring images during the conservative treatment period
2. Santucci RA, McAninch JW (2000) Diagnosis and management of renal trauma: past, present and future. J Am Coll Surg 191(4):443-451

3. Shuai WJ, You FS, Zhang W et al (2008) Image monitoring for an intraperitoneal bleeding model of pigs using electrical impedance tomography. Physiol Meas 29:217-225

4. Shuai WJ, You FS, Zhang HY et al (2009) Application of electrical impedance tomography for continuous monitoring of retroperitoneal bleeding after blunt trauma. Ann Biomed Eng 37:2373-2379

5. Xu CH, Dai M, You FS et al (2011) An optimized strategy for real-time hemorrhage monitoring with electrical impedance tomography. Physiol Meas $32: 585-598$

F. You $\cdot$ X. Shi $\cdot$ F. Fu $\cdot$ R. Liu

C. $\mathrm{Xu} \cdot \mathrm{X}$. Dong $(\bowtie)$

School of Biomedical Engineering, Fourth Military Medical University (FMMU), 710032 Xi'an, China e-mail: dongxiuzhen@fmmu.edu.cn

W. Shuai

Department of Medical Engineering, First Affiliated Hospital of 301 General Hospital, 100037 Beijing, China

H. Zhang - W. Zhang - T. Bao Department of Urology, TangDu Hospital, Fourth Military Medical University, 710038 Xi' an, China monitoring tool, could detect ARB early and enable timely intervention.

Acknowledgments This work was partially supported by the National Key R\&D Program of China under grant no. 2012BAI20B02 and the Natural Science Foundation of China (NSFC) under grant nos. 51177166 and AWS11C010.

Conflicts of interest None.

Open Access This article is distributed under the terms of the Creative Commons
Attribution Noncommercial License which permits any noncommercial use, distribution, and reproduction in any medium, provided the original author(s) and the source are credited.

\section{References}

1. Moore EE, Shackford SR, Pachter HL et al (1989) Organ injury scaling: spleen, liver and kidney. J Trauma 29:1664-1666 